\title{
Akt inhibitor MK-2206 enhances the effect of cisplatin in gastric cancer cells
}

\author{
KAIXIONG TAO ${ }^{1 *}$, YUPING YIN $^{1 *}$, QIAN SHEN $^{2}$, YING CHEN $^{1}$, RUIDONG LI $^{1}$, WEILONG CHANG ${ }^{1}$, \\ JIE BAI $^{1}$, WEIZHEN LIU ${ }^{1}$, LIANG SHI ${ }^{1}$ and PENG ZHANG ${ }^{1}$ \\ ${ }^{1}$ Department of General Surgery, Union Hospital; ${ }^{2}$ Department of Oncology, Tongji Hospital, Tongji Medical College, \\ Huazhong University of Science and Technology, Wuhan, Hubei 430022, P.R. China
}

Received November 25, 2015; Accepted January 27, 2016

DOI: $10.3892 /$ br.2016.594

\begin{abstract}
The phosphoinositide 3-kinase/Akt pathway activation commonly occurs in various types of human cancer and has an important role in chemoresistance. Combination of traditional chemotherapy drugs and molecular-targeted agents is a promising strategy for cancer therapy, which has shown enhanced cytotoxicity and lower drug resistance. The present study found that the Akt inhibitor, MK-2206, can increase the effect of cisplatin in the gastric cancer cell line AGS, which has higher Akt phosphorylation, but exhibited a poor combination effect in MKN-45 and MGC-803 cells, which have limited Akt activation. The MTT assay demonstrated that sequential treatment of cisplatin, followed by the Akt inhibitor, MK-2206, caused a synergistic effect of proliferation inhibition, and the apoptosis assay by propidium iodide/fluorescein isothiocyanate staining also showed that combination treatment induced more apoptosis compared to the monotherapy groups. Using western blot analysis, MK-2206 was shown to significantly suppress the phosphorylation of Akt (Ser473), however, the expression of total Akt remained the same, and the combination treatment also increased the expression of cleaved poly adenosine diphosphate ribose polymerase, which contributed to apoptosis.
\end{abstract}

\section{Introduction}

Gastric cancer is a type of common and fatal digestive malignant disease, particularly in eastern Asia (1), which

Correspondence to: Dr Peng Zhang, Department of General Surgery, Union Hospital, Tongji Medical College, Huazhong University of Science and Technology, 1277 Jiefang Road, Wuhan, Hubei 430022, P.R. China

E-mail: zhangpengwh@hust.edu.cn

*Contributed equally

Key words: phosphoinositide 3-kinase/Akt pathway, MK-2206, cisplatin, gastric cancer has a tendency to occur as metastasis in the early stage. Despite the progress in earlier diagnosis and new therapeutic strategies, which improved the overall prognosis for gastric cancer patients, advanced gastric cancer remains a rigorous challenge for clinical staff, and the median overall survival is <1-year, and 5-year survival is $\sim 7 \%$ for metastasis gastric cancer patient (2). Chemotherapy is an extremely important therapeutic strategy for advanced gastric cancer; however, chemotherapy resistance is a severe obstacle (3), particularly for patients who make progression or reoccur subsequent to finishing their first-line treatment, and these patients are rarely suitable for additional treatment as tumor cells show a poor response to any cytotoxic drugs or drug combinations (4).

To overcome the dilemma induced by chemotherapy resistance, numerous molecular-targeted agents have been designed and considered as new therapeutic strategies for several malignant diseases $(5,6)$, such as trastuzumab for Her-2 positive in breast cancer and gastric cancer $(7,8)$, and a number of other attractive molecular-targeted agents have also been merged and have shown promising data in preclinical trials (9-11).

The phosphoinositide 3-kinase (PI3K)/Akt pathway dysregulation has been widely reported in different types of malignant diseases $(12,13)$, such as the PIK3CA gene amplification or activation mutation, overactivation of the downstream molecule Akt, and loss of tumor suppressor phosphatase and tensin homolog (14). In gastric cancer, the abnormalities of the PI3K/Akt pathway are considered to have an important role in carcinogenesis, proliferation and resistance to chemotherapy drugs $(15,16)$. Additionally, as reported in a previous study, the treatment of conventional chemotherapy drugs can induce further activation of the PI3K/Akt pathway in a number of solid cancers (17), which may lead to the occurrence of acquired drug resistance. Therefore, we hypothesize that Akt inhibition may increase the efficiency of chemotherapy and reverse the drug resistance.

MK-2206 is a highly selective oral Akt inhibitor, which has a higher inhibition effect on Akt1 and Akt2, compared to Akt3. In vitro, MK-2206 is found to have a synergistic effect with conventional chemotherapy agents in ovarian cancer and osteosarcoma $(18,19)$. However, to the best of our knowledge, there are no previous studies evaluating the combination effect of cisplatin and MK-2206 in gastric cancer. 


\section{Materials and methods}

Cell culture. Gastric cancer cell lines AGS, MGC-803 and MKN-45 were purchased from the Cell Bank of China (Shanghai, China), and all these three cell lines were grown in RPMI-1640 (Thermo Fisher Scientific, Inc., Waltham, MA, USA) supplemented with $10 \%$ fetal bovine serum (Gemini Scientific, West Sacramento, CA, USA) at $37^{\circ} \mathrm{C}$ in a humidified atmosphere with $5 \% \mathrm{CO}_{2}$, and the culture medium was routinely changed every 3 days.

Drug sensitivity assay. Cell were seeded in 96-wells plate with a density of 5,000 cells/well, treated with cisplatin (Sigma-Aldrich, Inc., St. Louis, MO, USA) for 24 h, followed by the Akt inhibitor, MK-2206 (Selleck Chemicals, Houston, TX, USA), for another $48 \mathrm{~h}$. The monotherapy control groups were treated with cisplatin for $24 \mathrm{~h}$ followed by dimethyl sulfoxide (DMSO) (Sigma-Aldrich, Inc.) for another $48 \mathrm{~h}$, or were treated with MK-2206 alone for $72 \mathrm{~h}$, and the culture medium in each well was discharged and $20 \mu 1$ 3-(4,5-dimethylthiazol-2-yl)-2,5-diphenyltetrazolium bromide (MTT) (Sigma-Aldrich, Inc.) was added. The cells were cultured for another $4 \mathrm{~h}$, MTT in each well was removed and $150 \mu \mathrm{l}$ DMSO was added to each well and was cultured for another 5 min to dissolve the crystal at $37^{\circ} \mathrm{C}$. The absorbance was measured by microplate reader at $490 \mathrm{~nm}$ (EXI800; BioTek, WInooski, VT, USA).

Apoptosis assay by flow cytometry. Subsequent to seeding the AGS gastric cancer cells in 6-well plates with a density of $2 \times 10^{5}$ cells/well, the cells were treated with cisplatin for $12 \mathrm{~h}$, followed by MK-2206 for $24 \mathrm{~h}$, and the monotherapy groups were treated with cisplatin for $12 \mathrm{~h}$ followed by DMSO for another $24 \mathrm{~h}$ or MK-2206 alone for $24 \mathrm{~h}$. The control group was treated with DMSO alone. Following the harvesting of cells in each well, staining was performed with the Annexin V/propidium iodide (PI) kit (Nanjing Keygen Biotechnology, Nanjing, China) according to the manufacturer's protocol, and $\mathrm{PI} /$ fluorescein isothiocyanate two-dimensional flow cytometric analysis was performed.

Western blot analysis. The gastric cancer AGS cells were treated with cisplatin for $12 \mathrm{~h}$, followed by MK-2206 for $24 \mathrm{~h}$, and the cells were washed by phosphate-buffered saline twice, harvested and lysed in radioimmunoprecipitation assay buffer (Sigma-Aldrich, Inc.) with protease inhibitors. Cell lysates containing $50 \mu \mathrm{g}$ of protein were resolved in sodium dodecyl sulfate-polyacrylamide gel electrophoresis (SDS-PAGE), transferred onto a polyvinylidene fluoride filter, and probed with the antibodies of total Akt [rabbit monoclonal antibody (mAb); 1:1,000; Cell Signaling Technology, Danvers, MA, USA), p-Akt (Ser473; rabbit mAb; 1:1,000, Cell Signaling Technology), cleaved polyadenosine diphosphate ribose polymerase (PARP) (rabbit mAb; 1:1,000, Cell Signaling Technology) and glyceraldehyde 3-phosphate dehydrogenase (GAPDH) (rabbit mAb, 1:4,000; Sanying Biotechnology, Wuhan, China). Primary antibodies were detected with the secondary antibodies of goat anti-rabbit, using enhanced chemiluminescence (1:3,000; Beijing Beyotime Biology, Beijing, China).
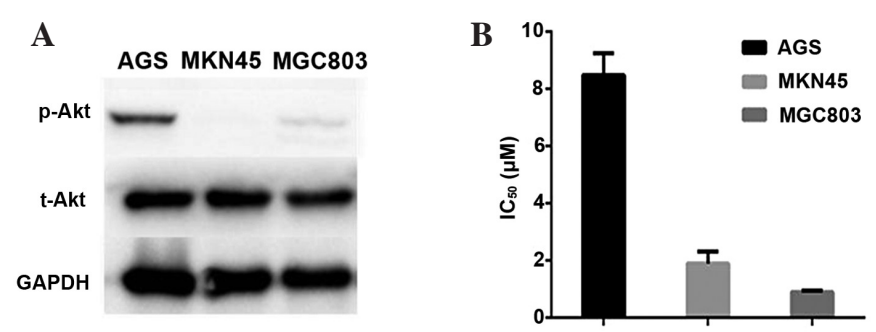

Figure 1. Expression of p-Akt (Ser473) and half maximal inhibitory concentration $\left(\mathrm{IC}_{50}\right)$ of cisplatin in gastric cancer cell lines. (A) The results of western blot analysis showed that the expression of p-Akt (Ser473) was highest in the gastric cancer AGS cell line. (B) From the results of the MTT assay, the $\mathrm{IC}_{50}$ of cisplatin in the AGS cells was also the highest in the three cell lines. The mean $\mathrm{IC}_{50}$ for AGS, MKN-45 and MGC-803 cells were 8.48, 1.89 and $0.89 \mu \mathrm{M}$.

Statistical analysis. Results were calculated as mean \pm standard deviation. Two-sided t-test was used to detect statistical significance, and $\mathrm{P}<0.05$ was considered to indicate a statistically significant difference.

\section{Results}

Gastric cancer cell line with the higher Akt phosphorylation is more resistant to cisplatin. To detect whether Akt phosphorylation has a role in drug resistance to cisplatin in gastric cancer, western blot analysis was performed to detect the level of p-Akt (Ser473) in three gastric cancer cell lines, AGS, MGC-803 and MKN-45. The level of p-Akt in AGS cells was higher compared to MGC-803 and MKN-45 cells, and the MTT assay was used to determine the effect of cisplatin in these three cell lines (Fig. 1A). The half maximal inhibitory concentration $\left(\mathrm{IC}_{50}\right)$ of cisplatin for each cell line was calculated. The $\mathrm{IC}_{50}$ of AGS was almost twice as high as for MGC-803 and MKN-45 (Fig. 1B), which may indicate that Akt phosphorylation can increase the resistance to cisplatin in gastric cancer cells.

Akt inhibitor MK-2206 increases the effect of cisplatin with a sequence-dependant manner in gastric cancer cells. As Akt phosphorylation may have a role in the resistance to cisplatin, we hypothesized that Akt inhibition may increase the effect of cisplatin in gastric cancer cells. To confirm this hypothesis, the Akt inhibitor MK-2206 was used, and in a previous study, three sequences have been used to detect the combination effect of MK-2206 and traditional chemotherapy drugs in cancer: Traditional drugs followed by MK-2206, MK-2206 prior to traditional drugs, and MK-2206 and traditional drugs simultaneously, and it has been reported that traditional drugs followed by MK-2206 showed a good effect, and the effect of other two sequences were limited $(18,19)$. MK-2206 can increase the effect of cisplatin in high p-Akt activation gastric cancer AGS cells, but the combination effect was poor in the other two gastric cancer cells with lower p-Akt (Fig. 2).

MK-2206 can increase the apoptosis induced by cisplatin in gastric cancer cell line AGS. The combination of MK-2206 and cisplatin lead to a synthetic cytotoxic effect in the higher Akt phosphorylation gastric cancer cell line, AGS; however, 

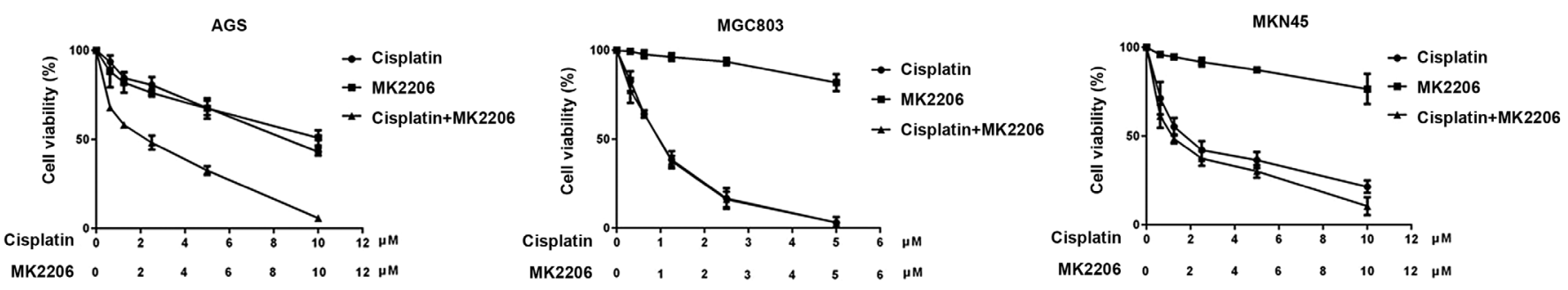

Figure 2. Effect of the cisplatin and MK-2206 combination in the gastric cancer cell lines, AGS, MGC-803 and MKN-45. Following treatment with cisplatin for $24 \mathrm{~h}$, followed with MK-2206 for another $48 \mathrm{~h}$, the results of the MTT assay showed that MK-2206 can significantly increase the effect of cisplatin in AGS cells $(\mathrm{P}<0.05)$, but MK-2206 showed a slight combination effect in the other two cell lines.
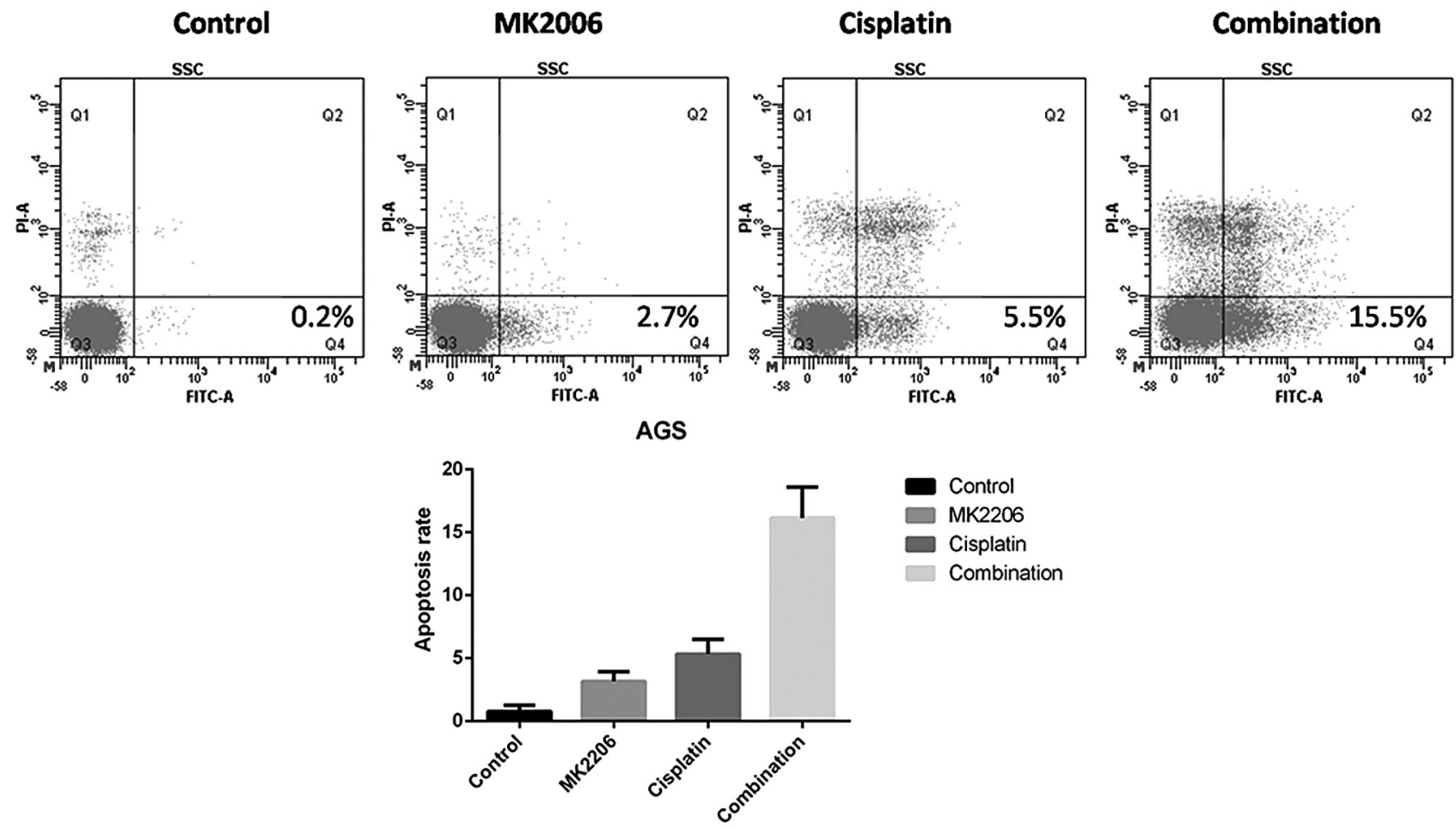

Figure 3. Effect of the cisplatin and MK-2206 combination on the apoptosis in AGS cells. The mean apoptosis rate in the combination groups was $16.17 \%$, which was higher compared to the MK-2206 and cisplatin monotherapy groups (3.17 and 5.33\%).

the combination showed a slight effect in the other two gastric cancer cell lines, which have a low level of Akt phosphorylation. To investigate whether MK-2206 can also increase the apoptosis induced by cisplatin, AGS cells were treated as aforementioned, and were subjected to Annexin V/PI staining. Sequential treatment of AGS cells with $10 \mu \mathrm{M}$ cisplatin for $12 \mathrm{~h}$ followed by $10 \mu \mathrm{M}$ MK-2206 for a further $24 \mathrm{~h}$ induced more apoptosis compared to the monotherapy groups (16.17\% in the combination group, $3.17 \%$ in the MK-2206 group and $5.33 \%$ in the cisplatin group) (Fig. 3).

MK-2206 inhibits the expression of $p$-Akt and increases the expression of cleaved PARP in the gastric cancer AGS cell line. MK-2206, as a potent Akt inhibitor, significantly decreased the Akt phosphorylation and activation. Subsequently, the effect of the potential inhibition of the Akt pathway by cisplatin and MK-2206 combination was analyzed. The results of western blot analysis (Fig. 4) showed that the combination can markedly decrease the p-Akt

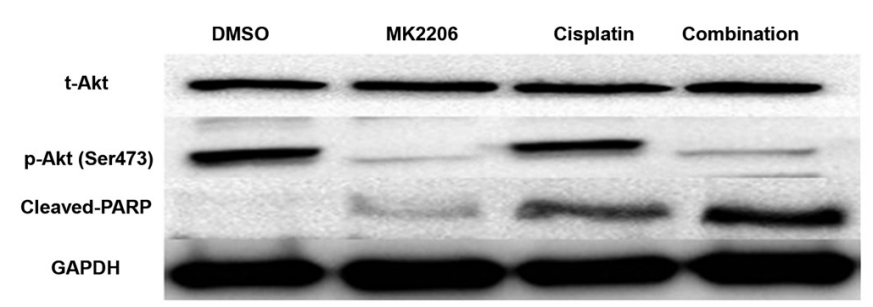

Figure 4. Effect of cisplatin and MK-2206 combination on the expression of total Akt, p-Akt and cleaved poly adenosine diphosphate ribose polymerase (PARP). MK2206 can significantly inhibit the expression of p-Akt (Ser473), and there was no difference in the level of total Akt in these four groups. In the combination group, the level of cleaved PARP was higher compared to the other three groups. DMSO, dimethyl sulfoxide.

(Ser473) in AGS cells, compared with control and cisplatin monotherapy groups; however, the total Akt remained stable in all groups. PARP is recognized as a marker of apoptosis, so therefore the effect of the combination on the expression of cleaved PARP was examined. In the untreated group, the 
expression of cleaved PARP was limited, and the combination group induced more expression of cleaved PARP in contrast to the cisplatin or MK-2206 alone groups, and this was in accordance with the results of the MTT and apoptosis assays.

\section{Discussion}

The limited effectiveness of chemotherapy drugs can be attributed to several reasons, such as checkpoints that arrest the cell cycle to allow DNA damage repair, or activation of certain signaling pathways to help the cancer cell escape from the cytotoxicity induced by numerous drugs $(20,21)$. Therefore, targeting survival pathways may be logically considered as a good strategy for increasing the chemotherapeutic effect in cancer cells $(22,23)$.

The PI3K/Akt pathway has been widely investigated, and was reported to participate in several biological and pathological responses in numerous types of cancer, including gastric cancer. Numerous molecules targeting the PI3K/Akt pathway have been designed (24), MK-2206 is one of these agents, although the monotherapy effect of MK-2206 is limited in a number of cancers, and certain studies have reported that it showed a good therapeutic effect when combined with other traditional chemotherapy drugs (25).

The present study evaluated the benefit of the combination of cisplatin and MK-2206 in gastric cancer cell lines, and detected the effect of the Akt inhibitor MK-2206 on gastric cancer cell lines alone and in combination of cisplatin. The monotherapy effect of MK-2206 was not significant in the three cell gastric cancer cell lines; however, when treating the AGS cells with cisplatin followed by MK-2206, an increase in apoptosis and proliferation inhibition was induced in gastric cancer cells. Western blot analysis also showed that the expression of p-Akt (Ser473) was significantly blocked in the combination group, and consistently, the level of the apoptosis marker, cleaved PARP, was higher compared to the monotherapy groups.

The present study has certain limitations. The number of gastric cancer cell lines used in the experiments is limited, there is no in vivo experiment evaluation and the mechanisms for the effect remain to be elucidated. However, the data appear promising, and may be helpful for high Akt phosphorylation gastric cancer patients.

\section{Acknowledgements}

The present study was sponsored by a grant from the National Nature Science Foundation of China (no. 81172294).

\section{References}

1. Siegel RL, Miller KD and Jemal A: Cancer statistics, 2015. CA Cancer J Clin 65: 5-29, 2015.

2. Power DG, Kelsen DP and Shah MA: Advanced gastric cancer - slow but steady progress. Cancer Treat Rev 36: 384-392, 2010.

3. Marin JJ,Al-Abdulla R, Lozano E, Briz O, Bujanda L, Banales JM and Macias RI: Mechanisms of resistance to chemotherapy in gastric cancer. Anticancer Agents Med Chem: Aug 3, 2015 (Epub ahead of print).
4. Kim SM and Park SH: Chemotherapy beyond second-line in advanced gastric cancer. World J Gastroenterol 21: 8811-8816, 2015.

5. Covell LL and Ganti AK: Treatment of advanced thyroid cancer: Role of molecularly targeted therapies. Target Oncol 10: 311-324, 2015.

6. Kito Y and Yamazaki K: Targeted therapies for metastatic colorectal cancer. Nihon Rinsho 73: 1384-1390, 2015 (In Japanese).

7. Wahler J and Suh N: Targeting HER2 Positive breast cancer with chemopreventive agents. Curr Pharmacol Rep 1: 324-335, 2015.

8. Chua C, Tan IB, Yamada Y, Rha SY, Yong WP, Ong WS Tham CK, Ng M, Tai DW, Iwasa S, et al: Phase II study of trastuzumab in combination with S-1 and cisplatin in the firstline treatment of human epidermal growth factor receptor HER2-positive advanced gastric cancer. Cancer Chemother Pharmacol 76: 397-408, 2015.

9. Bao Z, Cao C, Geng X, Tian B, Wu Y, Zhang C, Chen Z, Li W, Shen $\mathrm{H}$ and Ying S: Effectiveness and safety of poly (ADP-ribose) polymerase inhibitors in cancer therapy: A systematic review and meta-analysis. Oncotarget: Sep 22, 2015 (Epub ahead of print).

10. Abu-Khalaf MM, Baumgart MA, Gettinger SN, Doddamane I, Tuck DP, Hou S, Chen N, Sullivan C, Lezon-Geyda K, Zelterman D, et al: Phase 1b study of the mammalian target of rapamycin inhibitor sirolimus in combination with nanoparticle albumin-bound paclitaxel in patients with advanced solid tumors. Cancer 121: 1817-1826, 2015.

11. Brana I, Berger R, Golan T, Haluska P, Edenfield J, Fiorica J, Stephenson J, Martin LP, Westin S, Hanjani P, et al: A parallelarm phase I trial of the humanised anti-IGF-1R antibody dalotuzumab in combination with the AKT inhibitor MK-2206, the mTOR inhibitor ridaforolimus, or the NOTCH inhibitor MK-0752, in patients with advanced solid tumours. Br J Cancer 111: 1932-1944, 2014.

12. Faes $\mathrm{S}$ and Dormond O: PI3K and AKT: Unfaithful partners in cancer. Int J Mol Sci 16: 21138-21152, 2015.

13. Guo H, German P, Bai S, Barnes S, Guo W, Qi X, Lou H, Liang J, Jonasch E, Mills GB, et al: The PI3K/AKT pathway and renal cell carcinoma. J Genet Genomics 42: 343-353, 2015.

14. Liu P, Cheng H, Roberts TM and Zhao JJ: Targeting the phosphoinositide 3-kinase pathway in cancer. Nat Rev Drug Discov 8: 627-644, 2009.

15. Shi J, Yao D, Liu W, Wang N, Lv H, Zhang G, Ji M, Xu L, He N, Shi B, et al: Highly frequent PIK3CA amplification is associated with poor prognosis in gastric cancer. BMC Cancer 12: 50, 2012.

16. Matsuoka T and Yashiro M: The role of PI3K/Akt/mTOR signaling in gastric carcinoma. Cancers (Basel) 6: 1441-1463, 2014.

17. Parsons CM, Muilenburg D, Bowles TL, Virudachalam S and Bold RJ: The role of Akt activation in the response to chemotherapy in pancreatic cancer. Anticancer Res 30: 3279-3289, 2010.

18. Lin YH, Chen BY, Lai WT, Wu SF, Guh JH, Cheng AL and Hsu LC: The Akt inhibitor MK-2206 enhances the cytotoxicity of paclitaxel (Taxol) and cisplatin in ovarian cancer cells. Naunyn Schmiedebergs Arch Pharmacol 388: 19-31, 2015.

19. Duan L, Perez RE, Hansen M, Gitelis S and Maki CG: Increasing cisplatin sensitivity by schedule-dependent inhibition of AKT and Chk1. Cancer Biol Ther 15: 1600-1612, 2014.

20. Riquelme I, Saavedra K, Espinoza JA, Weber H, García P, Nervi B, Garrido M, Corvalán AH, Roa JC and Bizama C: Molecular classification of gastric cancer: Towards a pathwaydriven targeted therapy. Oncotarget 6: 24750-24779, 2015.

21. Benada J and Macurek L: Targeting the checkpoint to kill cancer cells. Biomolecules 5: 1912-1937, 2015.

22. Nicolini A, Ferrari P, Kotlarova L, Rossi G and Biava PM: The PI3K-AKt-mTOR pathway and new tools to prevent acquired hormone resistance in breast cancer. Curr Pharm Biotechnol 16: 804-815, 2015.

23. Pandey R and Kapur R: Targeting phosphatidylinositol-3-kinase pathway for the treatment of Philadelphia-negative myeloproliferative neoplasms. Mol Cancer 14: 118, 2015.

24. Zhang L, Wu J, Ling MT, Zhao L and Zhao KN: The role of the $\mathrm{PI}$ KK/Akt/mTOR signalling pathway in human cancers induced by infection with human papillomaviruses. Mol Cancer 14: 87, 2015.

25. Almhanna K, Cubitt CL, Zhang S, Kazim S, Husain K, Sullivan D, Sebti S and Malafa M: MK-2206, an Akt inhibitor, enhances carboplatinum/paclitaxel efficacy in gastric cancer cell lines. Cancer Biol Ther 14: 932-936, 2013. 